\title{
Asma en el paciente pediátrico
}

Asthma in children

Andrés Benincore Robledo*, Lidia María Prado López **

\section{RESUMEN}

El asma es una enfermedad respiratoria crónica y heterogénea que afecta a más de 250 millones de personas en todo el mundo. Su origen es multifactorial y está determinada por factores predisponentes y desencadenantes que resultan en un fenotipo específico. La forma de presentación más común en niños es el alérgico, de inicio temprano y desencadenada por alérgenos. La sospecha inicial es clínica, con la triada clásica de tos, sibilancias y dificultad respiratoria desencadenada por factores ambientales específicos, empeora con cuadros infecciosos y varía con el tiempo. En mayores de 6 años, la confirmación diagnóstica se hace con pruebas de función respiratoria. En menores de esta edad el diagnóstico es complejo debido a las sibilancias asociadas a infecciones y broncoespasmo post-infeccioso, por lo que se han desarrollado herramientas para ayudar a diferenciarlo. El tratamiento se determinará según la clasificación por severidad. La base es el esteroide inhalado, y el objetivo es encontrar la dosis mínima necesaria para controlar los síntomas siguiendo el esquema escalonado de tratamiento, en el que se puede combinar con otros medicamentos, según el control que se tenga. Es esencial llevar un seguimiento estrecho, con citas cada tres meses para subir o bajar en la escalera según sea necesario. El uso correcto de inhaladores es primordial para lograr buen control. En caso de perderse éste puede sobrevenir una crisis. El conocimiento del paciente y sus padres sobre cómo identificarla y tratarla en su inicio, son esenciales

\footnotetext{
* Especialista en Pediatría, Residente de 2do Año de Neumología Pediátrica Instituto Nacional de Pediatría (INP) - México.

** Especialista en Pediatría, Residente de 1er año Neumología Pediátrica, INP.

Dirigir correspondencia a: abenincore@gmail.com

Recibido: 20 de diciembre 2017 Aprobado: 10 de febrero 2018
}

para evitar complicaciones.

\section{PALABRAS CLAVE}

Asma, diagnóstico, etiología, tratamiento farmacológico

\section{ABSTRACT}

Asthma is a chronic and heterogeneous disease that affects more than 250 million people around the world. In children, it has a prevalence of $13.7 \%$. It has a multifactorial origin with a genetic base, and its clinical expression will depend on the predisposing and triggering factors that result in a specific phenotype. Allergic phenotype is most common in children, and has an earlier onset. Diagnosis is suspected by the clinical triad of cough, wheezing and distressed breathing which can be triggered by an allergen, exercise or infection, and vary in time and intensity. In children 6 years and older confirmation can be obtained by pulmonary function tests, which will reveal reversible airway obstruction. In younger children the diagnosis might not be as easy due to the many factors that can trigger cough and wheezing other than asthma (for example virus-induced wheezing). Tools (mAPI) have been developed to help differentiate between asthma and other causes of wheezing. Treatment is based on inhaled steroids, and the aim of the physician must be to find the minimum necessary dose to maintain control of symptoms. Treatment can be escalated or lowered according to the "treatment ladder", and second medications (LABA) can be added if needed. Inhaler technique is as important as the medication itself to achieve control, and must be checked frequently. If control is lost, an asthma crisis might occur. Caregiver's knowledge on identifying and treating the crisis is essential to prevent complications. 


\section{KEYWORDS}

Asthma, diagnosis, drug therapy, etiology.

\section{INTRODUCCIÓN}

El asma es la enfermedad respiratoria crónica más común en la población pediátrica. Puede iniciar en los primeros años de vida, y desencadenarse por infecciones virales o factores ambientales. El control de los síntomas es posible si se hace un diagnóstico oportuno y se da un tratamiento adecuado, mejorando así la calidad de vida tanto del paciente como de sus cuidadores.

\section{EPIDEMIOLOGÍA}

El asma es un problema de proporciones pandémicas. Se estima que afecta entre 235 y 300 millones de personas en todo el mundo, ${ }^{(1,2)}$ y hasta al $7 \%$ de la población en México, (1) es decir a más de 8 millones de personas. La población pediátrica es la más afectada, con una prevalencia global de entre $11.6-13.7 \%$, y mortalidad que va entre 0.03 y $0.4 \%$. (1) Según la Dirección General de Información en Salud (DGIS) en México durante el 2013 el $20 \%$ de las hospitalizaciones por enfermedades respiratorias se debieron a asma. ${ }^{(1)}$

El impacto económico que tiene la enfermedad, tanto en los hogares como a nivel gubernamental, es altísimo. En un estudio realizado en México en el 2006 se estimó que en las familias con un niño asmá-tico hasta el $19 \%$ del ingreso se invierte en la enfermedad (atención médica, medicamentos, hospitalizaciones). ${ }^{(3)}$ Además, a esto hay que sumarle el costo del ausentismo laboral de los padres, que se calcula entre 2-5 días al año. ${ }^{(3)}$

\section{ETIOPATOGENIA}

El asma es una enfermedad heterogénea caracterizada por inflamación crónica de la vía aérea. ${ }^{(4,5)}$ Tiene una base genética y un origen multifactorial en el que intervienen diversos factores ambientales. La interacción entre factores predisponentes (genéticos) y desencadenantes (alérgenos, agentes infecciosos, contaminantes ambientales, irritantes, estrés emocional, entre otros) da origen a la expresión clínica del asma, que no es igual en todos los individuos. A esto se le conoce como fenotipo. ${ }^{(6,7)}$

El fenotipo más común en niños es el asma alérgica. Es de inicio temprano, y está ocasionada por una respuesta inmunológica específica hacia un alérgeno que puede o no ser mediada por IgE. Los linfocitos (generalmente Th2) ante la identificación de un alérgeno (polvo, ácaros, polen, etc) producen diversas interleucinas (IL-4, IL-5, IL-9, IL-13), que a su vez generan la degranulación de eosinófilos. Estos secretan diversas sustancias pro-inflamatorias (histamina, leucotrienos, prostaglandinas, proteasas) que ocasionan broncoespasmo, edema e hipersecreción de moco, causantes de la obstrucción característica del asma. ${ }^{(5,8)}$ En un inicio los cambios son reversibles, por lo que en períodos libres de estímulos la inflamación puede disminuir tanto que la obstrucción es mínima y no hay síntomas. ${ }^{(9,10)}$

Los estímulos constantes (infecciones virales, exposición continua al alérgeno, contaminación ambiental, humo de cigarro o leña) propagan y perpetúan la inflamación, y con ésta, el daño a la vía aérea. Si no hay un control adecuado, eventualmente se produce remodelamiento de la mucosa, lo que deriva en obstrucción fija e irreversible con la consecuente disminución de la función pulmonar. ${ }^{(8,11,12)}$

El asma alérgica se da en un contexto de atopia, con antecedentes positivos ya sea en el paciente 0 en familiares. Generalmente hay eosinofilia tanto en la biometría hemática como en el esputo. La respuesta a esteroides inhalados (CEI) es excelente. ${ }^{(4,5,13)}$ 
El asma no alérgica, por el contrario, se da generalmente en adultos. No hay antecedentes de atopia en la familia, y la inflamación de la vía aérea es predominantemente neutrofílica. La respuesta a CEI no es tan buena, por lo que hay dificultad para controlar los síntomas con el tratamiento habitual. (4,5) $^{-}$

\section{DIAGNÓSTICO}

La sospecha diagnóstica de asma en el paciente pediátrico se hace por clínica. Un patrón característico de signos y síntomas, junto con ciertos antecedentes tanto heredofamiliares como personales patológicos, sugieren la enfermedad..$^{(1,4,5)}$

Los datos clínicos característicos son tos, sibilancias y disnea. La tos generalmente es seca, pero puede ser productiva si el cuadro está acompañado por una infección de vía aérea superior. Tanto la tos como las sibilancias se presentan o agravan en las noches (despiertan al paciente) o temprano en las mañanas, y empeoran con el frío, polvo, humo de cigarro o cualquier otro irritante inhalado. $A$ veces se desencadenan con el ejercicio (o con el llanto intenso en lactantes). La disnea, o sensación de falta de aire, es difícilmente referida por los niños. Generalmente es detectada por sus cuidadores al notar que el niño juega menos que sus compañeros, evita la actividad física, o se cansa pronto al caminar (pide ser cargado constantemente). ${ }^{(4,5)}$

La característica principal de estos síntomas es que varían con el tiempo y en intensidad. Así, sibilancias constantes, que no mejoran o empeoran, y que persisten por mucho tiempo deben hacer pensar en diagnósticos diferenciales. ${ }^{(14)}$

En el interrogatorio es importante preguntar sobre antecedentes de asma en la familia, ya que familiares de primer grado con asma, en el contexto de un niño con la clínica mencionada previamente, hacen el diagnóstico muy probable. Antecedentes de eczema, rinitis alérgica, o alergias alimentarias ponen al paciente en un contexto de atopia, que también favorece el diagnóstico. ${ }^{(6)}$

En niños menores de 5 años el diagnóstico puede ser difícil ya que en ellos "no todo lo que silba es asma", por lo que se ha acuñado el término de sibilante recurrente en lactantes que han presentado $\geq 3$ episodios de sibilancias desde su nacimiento. ${ }^{(15)}$ La recurrencia de sibilancias puede ser secundaria a alteraciones estructurales (traqueobroncomalacia, compresiones vasculares, estenosis traqueal, masa o lesiones quísticas, tumores o linfadenopatías, cardiomegalia) o funcionales (asma, reflujo gastroesofágico patológico, aspiración, inmunodeficiencia, displasia, bronquiolitis obliterante, disfunción de cuerdas vocales, enfermedad pulmonar intersticial, cuerpo extraño). ${ }^{(16)}$

La tos y sibilancias son síntomas comunes en infecciones de vía respiratoria, especialmente virales. . $^{(4,17)}$ El término "sibilancias asociadas a infecciones" se usa en aquellos casos en los que el broncoespasmo se da sólo asociado a cuadros catarrales. Algunos virus (rinovirus y virus sincicial respiratorio) dejan daño a la mucosa de la vía aérea, ocasionando cuadros de broncoespasmo ante estímulos (ejercicio, irritantes, alérgenos) aun cuando el cuadro gripal ha desaparecido. A esto se le conoce como "hiperreactividad bronquial post-infecciosa", y puede confundir al médico en cuanto al diagnóstico de asma. Sin embargo, algunos de éstos niños desarrollarán asma eventualmente como consecuencia de la infección, por lo que se debe mantener vigilancia estrecha. ${ }^{(1,5,18)}$

La probabilidad de que las sibilancias sean secundarias a infección viral, y por lo tanto, transitorias, aumenta si los síntomas aparecen sólo durante cuadros gripales y hasta máximo 10 días posterior a éstos, si hay 3 o menos episodios al año, y si el niño se mantiene asintomático entre cada episodio. ${ }^{(14)}$ 
La dificultad de hacer diagnóstico de asma en este grupo etario es tal, que se han desarrollado herramientas para predecir la probabilidad de un niño de desarrollar la enfermedad. En el año 2000, Castro-Rodríguez propuso el uso del Índice Predictivo de Asma (API, por sus siglas en inglés) para identificar niños potencialmente en riesgo de padecer asma en edad escolar. Los niños deben presentar sibilancias frecuentes y tempranas durante los primeros 3 años de vida, y cumplir al menos uno de los dos criterios principales (padres asmáticos o eczema en el niño, ambos diagnosticados por médico) o dos de los tres criterios menores (rinitis alérgica diagnosticada por médico, sibilancias en periodos $\sin$ resfriados o eosinofilia $\geq 4 \%$ ). Si se cumplen los criterios, el paciente tiene 9.8 veces mayor riesgo de padecer asma a los 6 años, un valor predictivo positivo de $47.5 \%$ y valor predictivo negativo de $91.6 \%$. $^{(19)}$ Se han realizado modificaciones a dicho índice, conocido por sus siglas en ingles mAPI (Indice Predictivo de Asma modificado). mAPI está validada en niños de 3 años o menos, en la que se toman en agregaron criterios mayores y menores que se enumeran en la Tabla 1. ${ }^{(20,21)}$ (mAPI, recurso disponible en línea en https://www.mdcalc.com/modified -asthma-predictive-index-mapi). Con las modificaciones realizadas, el riesgo de padecer asma a los 6 años incrementó a 21 veces, ${ }^{(19)}$ y VPP $77 \% .{ }^{(5)}$ A pesar de lo anterior, el índice más utilizado es API, ya que la determinación de la eosinofilia periférica es más fácil y barato que las pruebas de alergía y es más confiable ya que hay variaciones por regiones de la sensibilización a alérgenos. ${ }^{(22)}$

Si la sospecha clínica es alta, es válido realizar una prueba terapéutica con CEI por 2 o 3 meses. En caso de presentar mejoría significativa de los síntomas, el niño deberá continuar con el tratamiento, siguiendo los lineamientos expuestos más adelante. En caso de no notar mejoría, se deberá suspender el medicamento, siempre manteniendo una vigilancia estrecha, con citas regulares a la consulta y datos de alarma a los cuidadores. Si los síntomas se agravan, se deberá reiniciar el esteroide y continuar el tratamiento como asmático. ${ }^{(4,5)}$

En mayores de 6 años es importante intentar documentar la obstrucción reversible de la vía aérea por medio de pruebas de función respiratoria. En caso de contarse con el recurso se deberá solicitar una espirometría basal y con broncodilatador previo al inicio del CEI, y otra posterior a 3-6 meses de iniciado el tratamiento. ${ }^{(1)}$ Con esto se confirmará el diagnóstico, y se tendrá un basal para darle seguimiento a la función pulmonar. Sin embargo, nunca debe retrasarse el manejo de un paciente con asma por obtener esta confirmación. ${ }^{(4)}$

El óxido nítrico es una molécula gaseosa que regula el tono vascular y bronquial, cuya concentración está incrementada en pacientes con inflamación eosinofílica de la vía aérea. La medición de la fracción exhalada de Óxido Nítrico (FENO) mayores a 35 ppb en niños está relacionada con buena respuesta a CEI. ${ }^{(23)}$ La eosinofília periférica también es un marcador de inflamación eosinofílica además de ser un marcador de riesgo de exacerbación. ${ }^{(24)}$

Otros métodos diagnósticos complementarios son las pruebas cutáneas (prick-test) y la IgE específica para alérgenos comunes. La radiografía de tórax será de utilidad cuando se tenga duda en el diagnóstico y se quieran descartar diagnósticos diferenciales. ${ }^{(4)}$

\section{CLASIFICACIÓN}

En la Guía Mexicana de Asma 2017 se sugiere clasificar inicialmente el asma de acuerdo a 4 aspectos:

1) El nivel de control, 2) Riesgo futuro de exacerbación, 3) Gravedad (intermitente versus persistente) y 4) Fenotipo. ${ }^{(5)}$ 
El asma se clasifica según la frecuencia (control) de los síntomas, como lo define GINA, evalúa interrogando al paciente o a cuidadores sobre los síntomas en las últimas 4 semanas, con cuatro ítems principales: síntomas durante el día, síntomas durante la noche (despertares), uso de medicamento de rescate (salbutamol), y limitación de la actividad física. Esto pondrá al paciente en uno de tres grupos: Controlado (si no hay alteración en ningún ítem), parcialmente controlado (con alteración de 1-2 ítems), o no controlado (alteración de 3-4 ítems).

Existen también recursos en línea como el Test de Control de Asma (ACT), validado en niños de 4 años en adelante, que permiten una rápida clasificación del control según el puntaje obtenido en el cuestionario (20-25 bien controlado, 16-19 parcialmente controlado, 5-15 mal controlado). ${ }^{(25)}$

El riesgo a futuro se determina según los factores que tiene el paciente que lo hace propenso a complicaciones a largo plazo. Tener un mal control de los síntomas (ACT menor a 20 puntos) eleva el riesgo de presentar una crisis de asma en los próximos 12 meses de manera considerable. El uso elevado de medicamento de rescate (salbutamol más de $1 \mathrm{vial} / \mathrm{mes}$ ), mala técnica o mal apego a los CEl, problemas socio-económicos, exposición a humo de tabaco u otros contaminantes, y comorbilidades como obesidad, rinitis alérgica o alergia alimentaria, son factores que entran en la categoría de "modificables", ya que por cambios en la conducta del paciente y cuidadores pueden controlarse. Los factores de riesgo "no-modificables" son: haber tenido una o más crisis severas de asma en los últimos 12 meses (que ameritara acudir a urgencias), y haber estado intubado por asma. (Ver tabla No. 2).

Después de confirmar el diagnóstico, es importante evaluar y clasificar al paciente para poder tomar una decisión en cuanto al tratamiento, recomendaciones, y seguimiento.

Después del manejo de mantenimiento y con la sintomatología estable se clasifica el asma basado en la gravedad según el "paso de tratamiento" mínimo necesario para mantener un buen control de la enfermedad o el valor de FEV1. Se clasifica en leve (paso 1 o 2, o VEF1>80), moderada (paso 3 o 60<VEF1 >80) o severa (paso 4 o 5, o VEF1<60). ${ }^{(26)}$ Para esto es necesario haber iniciado tratamiento, y haber intentado disminuir por lo menos un escalón antes de poder determinar el manejo adecuado para el paciente. Es por esto que la severidad no se puede determinar hasta por lo menos 3-6 meses de iniciado el medicamento controlador. ${ }^{(4,5)}$

\section{Evaluación del paciente en tratamiento}

Al detectar un paciente con mal control (ACT menor a 20 puntos), es necesario revisar que la técnica de administración de los medicamentos inhalados sea la correcta, que se está usando el medicamento apropiado a las dosis y posología indicada, mediante el dispositivo correcto según la edad, y que los factores modificables están controlados, antes de considerar subir de paso en la escala de tratamiento.

\section{TRATAMIENTO}

El objetivo del tratamiento del asma es lograr un adecuado control de los síntomas que permita mantener al paciente un nivel de actividad normal, así como minimizar los riesgos a futuro.

Los medicamentos en asma se dividen en 3 categorías: Los controladores (esteroides inhalados o $\mathrm{CEI}$ ) reducen la inflamación de la vía aérea y permiten el control de los síntomas, minimizando el riesgo de exacerbaciones (Ver tabla No. 3). Los de rescate (b2-agonistas de acción corta o SABA), 
producen broncodilatación casi inmediata, por lo que son de elección para el tratamiento de las exacerbaciones o crisis asmáticas.

Los medicamentos agregados (b2-agonistas de acción prolongada o LABA, antileucotrienos, anticolinérgicos) se usan como coadyuvantes cuando no se logra un control adecuado, ${ }^{(5,27)}$ y se pueden utilizar de manera conjunta con los CEI como controladores en los casos en los que éstos como monoterapia no son suficientes.

Los anticolinérgicos de acción prolongada, en concreto el tiotropio, son usados en casos de asma de difícil control en los que dosis altas de CEI y LABA no logran mantener al paciente asintomático. ${ }^{(5,21)}$ Es importante recordar que el uso de tiotropio no está aprobado en menores de 12 años, (21) aunque algunas guías lo mencionan como parte del tratamiento de asma grave en pacientes de 6-12 años, siempre bajo la estricta supervisión del especialista. ${ }^{(5)}$

El tratamiento del asma se encuentra organizado en un esquema escalonado ("pasos"), sobre el que se debe ir subiendo o bajando según la evolución clínica del paciente, con el fin de encontrar la dosis mínima necesaria para mantener al paciente asintomático..$^{(1,4,5)}$ (Ver Tabla No.4).

El paso 1 consiste en manejo de rescate solamente. No incluye medicamento controlador, por lo que se sugiere nunca iniciar con este paso.

El paso 2 consiste en $\mathrm{CEI}$ a dosis bajas como controlador, y b2-agonista de acción corta como medicamento de rescate. Es el paso para iniciar tratamiento sugerido por la mayoría de las guías. En adolescentes muy sintomáticos o con crisis asmática al momento del diagnóstico se puede iniciar con paso 3 a criterio del médico.
EI paso 3 va a variar según la edad del paciente. Las guías americanas (GINA) sugieren que en mayores de 5 años consiste en dosis bajas de CEI + LABA y en menores de 5 años dosis media de CEI. Sugerencia similar se encuentra en GUIMA pero con edad de corte de 4 años. ${ }^{(1,4,5)}$

El paso 4 en mayores de 5 años consiste en dosis medias de CEI + LABA. En menores de 5 años, la GINA recomiendan continuar dosis media de CEI y referir con especialista. La GUIMA sugiere en mayores de 4 años CEI a dosis media + LABA, y referencia con el especialista en caso de no lograr el control. El especialista en estos casos puede considerar el uso de tiotropio (tomando en cuenta las consideraciones mencionadas previamente con respecto a este medicamento), o de medicamentos biológicos, terapia dirigida contra anticuerpos específicos (omalizumab vs. IgE) o interleucinas (mepolizumab vs. IL-5) dependiendo de las características fenotípicas del paciente (asma alérgica o asma eosinofílica respectivamente). ${ }^{(5,21,22)}$

En menores de 4 años no está aprobado el uso de LABA, y los riesgos de usar CEI a dosis alta hacen que no se recomienden en este grupo de edad. ${ }^{(5,13)}$

Antes de subir un escalón se debe revisar la técnica de aplicación de los medicamentos inhalados, asegurarse que el paciente está teniendo un buen apego, corregir factores de riesgo modificables y realizar medidas no farmacológicas. ${ }^{(28)}$

Una vez se hace cambio en el tratamiento, se debe evaluar la respuesta en 3 meses. Si se logró el control y no existen factores de riesgo, se puede disminuir un paso en la escalera. ${ }^{(1,13,29,30)}$

\section{Uso correcto de Inhaladores}

Existen diferentes dispositivos para adminis- 
trar los medicamentos inhalados. Para pacientes pediátricos el de elección es el inhalador de dosis medida presurizado (pIDM), aunque en pacientes mayores (escolares y adolescentes) es posible usar los inhaladores de polvo seco. ${ }^{(13,31,32)}$

Es recomendable usar los pIDM con espaciador, sin importar la edad del paciente, ya que mejoran la entrega del medicamento a la vía aérea y minimizan los efectos secundarios locales de los CEI. El espaciador debe tener mascarilla facial en pacientes quienes no son capaces de coordinar de manera adecuada una inhalación por boca. En adolescentes es posible usar espaciadores con boquilla. ${ }^{(5,33)}$

\section{Tratamiento no Farmacológico}

Debido a la naturaleza multifactorial del asma, es muy importante tomar en cuenta el rol que los factores ambientales tienen tanto en el desarrollo, como en la falta de control y las exacerbaciones de la enfermedad. $(4,5,13,15,21)$

Por tal motivo, es también importante abordar éstos al momento de indicar el tratamiento, para que se hagan los cambios pertinentes en aquellos factores que son modificables, y se tomen precauciones para minimizar el daño de aquellos factores que no se pueden modificar.

El humo de tabaco es un conocido agente nocivo para la vía aérea, no solo para quien lo consume, sino para aquellos que están expuestos a éste de manera indirecta (fumadores pasivos). Es importante recomendar a los padres/cuidadores que se evite fumar dentro de la casa, carro, y otros lugares donde el paciente comúnmente habite, y mantenerlo alejado de zonas de fumadores. ${ }^{(5,13)}$

El ejercicio forma parte de un estilo de vida saludable. La natación, en específico, ha demostrado mejorar la función pulmonar. Es importante tener en cuenta que el broncoespasmo puede ser desencadenado por ejercicio, por lo que el paciente debe tener tratamiento adecuado y estar bien informado sobre qué hacer en caso de presentar una exacerbación.

La contaminación ambiental, factor no modificable, es un problema en la mayoría de grandes ciudades. La recomendación es evitar realizar actividades al aire libre cuando la calidad del aire es mala (altos índices de contaminación). ${ }^{(5,13)}$ La exposición a humo de leña, aún común en zonas rurales de Latinoamérica, es otro factor ambiental que empeora al paciente con asma, y que a largo plazo puede causar Enfermedad Pulmonar Obstructiva Crónica (EPOC). Se sugiere evitar la exposición crónica a este contaminante.

En casos de asma alérgica, es importante identificar el alérgeno y evitar la exposición a éste. Pelo de animales domésticos, ácaros, y polen de diversas plantas son los alérgenos más asociados a asma. ${ }^{(5,7,13)}$

\section{CRISIS ASMÁTICA}

El control del asma puede perderse por diversos factores (mal apego al tratamiento, infección viral, cambio de estación, etc), y los síntomas empeorar poco a poco hasta que el paciente presenta tos, disnea y sibilancias que ocasionan dificultad para respirar, o el paciente puede estar bien controlado, y de manera súbita tras un estímulo (exposición a alérgeno, irritantes, estrés emocional) puede presentar los síntomas en pocos minutos. A esto se le llama "crisis asmática". ${ }^{(5,13)}$

Existen diversas herramientas para clasificar la severidad de la crisis asmática, lo que ayuda al médico a evaluar la respuesta al tratamiento y determinar qué pacientes deber ser hospitalizados. (Ver Tablas No. 5 y No. 6). 


\section{Tratamiento}

El manejo de una crisis asmática debe iniciar al momento de detectarla, ya que entre más pronto se inicie el tratamiento, más fácil es que la crisis remita, y menor el riesgo de complicaciones. ${ }^{(34,35)}$

SABA inhalado administrado con espaciador es el tratamiento inicial de todas las crisis de asma. A una dosis de 200-600 mcg (de 2 a 6 puffs) cada 20 minutos hasta por 3 dosis, las crisis leves responderán de manera satisfactoria. En casos moderados o severos el uso de un anticolinérgico de acción rápida (bromuro de ipratropio) junto con SABA ha demostrado tener buen efecto.

En estos casos se deben iniciar esteroides sistémicos para controlar la inflamación y evitar complicaciones potencialmente fatales. Prednisona a 1-2 mg/kg/día (o equivalente) con dosis máxima de $40 \mathrm{mg} /$ día (50 $\mathrm{mg} / \mathrm{dí}$ en adolescentes y adultos), por 3-5 días, es el sugerido en las guías. ${ }^{(4,5,34)}$

El uso de oxígeno suplementario está recomendado para mantener SatO2 (por oximetría de pulso) por arriba de 92\% (>90\% si se encuentra por arriba de $2000 \mathrm{~m}$ sobre el nivel del mar).

Es importante reevaluar al paciente posterior a las tres dosis de SABA (o antes si existe un deterioro) con la misma escala que se utilizó al principio, con el fin de determinar si hubo mejoría con el tratamiento. En caso de no ser así, se deberán usar medicamentos de segunda línea para el tratamiento de la crisis. Sulfato de magnesio (MgSO4) intravenoso como dosis única está indicado en crisis moderadas o graves refractarias al manejo inicial, ${ }^{(4,5,33)}$ mientras que el uso de heliox (combinación de oxígeno y helio) para mejorar la administración del SABA o CEI pudiera ser efectivo en crisis severas, cuando se cuente con el recurso. ${ }^{(4)}$ El uso de metilxantinas (aminofilina) no está recomendado. ${ }^{(4,5)}$
El seguimiento del paciente posterior a una crisis de asma debe ser estrecho. Una consulta en la primera semana, y otra al mes, servirán para revisar nuevamente el control de los síntomas, el apego al tratamiento, y resolver cualquier duda que se tenga con respecto al uso del medicamento inhalado. ${ }^{(33,35)}$

\section{Caso Clínico}

"Paciente $X$ tiene 8 años. Fue diagnosticado con asma desde los 6 años. Usa como controlador fluticasona $50 \mathrm{mcg}$ cada 12 horas y salbutamol de rescate. En los últimos 6 meses ha dejado de hacer ejercicio por disnea. Se despierta algunas noches por accesos de tos. Ha tenido que acudir a urgencias por cuadros de dificultad respiratoria y sibilancias en 2 ocasiones. La madre refiere que ha olvidado darle el medicamento algunos días, y que nunca se lleva el salbutamol a la escuela".

El asma de este paciente se clasificaría como leve no controlada, con riesgo elevado a futuro de exacerbación por haber requerido manejo en urgencias en dos ocasiones en los últimos 6 meses.

Es asma leve porque necesita dosis bajas de CEI para mantener control de los síntomas. Sin embargo, es "No controlada" porque se encuentra muy sintomático, y esto se debe a un mal apego al tratamiento. El riesgo elevado a nuevas crisis de asma, y el antecedente de crisis en los últimos 6 meses, lo ponen en riesgo de obstrucción fija e irreversible de la vía aérea.

Lo recomendado en este caso es educar al paciente y sus familiares sobre la importancia del apego al tratamiento, verificar y aclarar dudas sobre el uso correcto de los medicamentos inhalados, y explicar las potencialmente mortales consecuencias de continuar con crisis asmáticas frecuentes. 
Es importante aclarar que no podemos clasificar al paciente como asma moderada o severa, debido a que los síntomas se controlan de manera satisfactoria con dosis bajas de esteroide inhalado. El problema en este caso fue la falta de apego al tratamiento, que lo llevaron al descontrol. Es por esto que no es necesario subir de paso en la escala de tratamiento por el momento.

\section{CONCLUSIONES}

- El asma es una enfermedad respiratoria crónica y heterogénea que afecta a más de 250 millones de personas en todo el mundo.

- El diagnóstico se basa en historia clínica sugestiva (tos, sibilancias, disnea), desencadenada por factores ambientales específicos (empeora con cuadros infecciosos), varía en el tiempo y respuesta al tratamiento.

- En mayores de 6 años, la confirmación diagnóstica se hace con pruebas de función respiratoria (espirometría basal y con broncodilatador), que evidencian una obstrucción reversible de la vía aérea. En menores de esta edad el diagnóstico es complejo debido a diagnósticos diferenciales, por lo que se han desarrollado herramientas para ayudar a diferenciarlos (mAPI).

- El tratamiento se determina según la clasificación por severidad y grado de control de los síntomas. El tratamiento inicial en un paciente que nunca ha tenido manejo inhalado se determina por la clínica que presenta al momento del diagnóstico. Una vez iniciado el manejo, se debe re-evaluar, y según la respuesta al tratamiento se puede determinar su SEVERIDAD (cuánto $\mathrm{CEl}$ necesita para mantener el control).

- La primera causa de "asma no controlada" es mal apego al tratamiento, y no significa agravamiento del asma. Una vez que se descarta mal apego o mal uso de los medicamentos como causa de descontrol, entonces se sube en la escalera de tratamiento y se re-clasifica la severidad (a moderada o severa). La severidad es dinámica, y puede aumentar o disminuir con el tiempo.

- El conocimiento del paciente y sus padres sobre cómo identificar las crisis y tratarlas en su inicio, son esenciales para evitar complicaciones.

Tabla No. 1: Indice Predictivo de Asma modificado (mAPI) en menores de 3 años.

\begin{tabular}{|c|}
\hline $\begin{array}{c}\text { Requisito en todos los pacientes: } \\
\text { y un criterio mayor o dos criterios menores }\end{array}$ \\
\hline Criterios Mayores \\
\hline - Padre con asma \\
- Dermatitis atópica (diagnosticada por médico) \\
- Sensibilidad a aero-alérgenos (prick test o lgE específica positivos) \\
\hline Criterios Menores \\
\hline - Sibilancias no relacionadas con cuadros gripales \\
- Eosinofilos $\geq 4 \%$ en sangre \\
\hline
\end{tabular}

Adaptado de: Chang TS, Lemanske RF, Guilbert TW, et al. Evaluation of the Modified Asthma Predictive Index in High-Risk Preschool Children. J Allergy Clin Immonological Pract. 2013;1(2):1-12. doi:10.1016/j.jaip.2012.10.008 
Tabla No. 2: Factores de riesgo para exacerbaciones.

\begin{tabular}{|l}
\hline Modificables \\
- Síntomas no controlados de asma \\
- Uso de $\geq 1$ vial de SABA al mes \\
- Mal uso de esteroide inhalado (mala técnica, mal apego) \\
- Exposición a humo de tabaco (o de leña) \\
- Problemas socio-económicos \\
\hline No Modificables \\
- Haber sido intubado por una crisis de asma \\
- $\geq 1$ exacerbación que requiriera manejo en urgencias en los últimos \\
\end{tabular}

Adaptada de: Global Initiative for Asthma. Global Strategy for Astham Management and Prevention, 2018.

Tabla 3: Dosis de esteroides inhalados (CSI).

\begin{tabular}{|c|c|c|c|}
\hline \multicolumn{4}{|c|}{ Corticoesteroides inhalados (CSI) } \\
\hline \multicolumn{4}{|c|}{ Adultos y Adolescentes ( $\geq 12$ años) } \\
\hline \multirow[t]{2}{*}{ Medicamentos } & \multicolumn{3}{|c|}{ Dosis diaria } \\
\hline & \multirow{2}{*}{$100-200$} & Media & Alta \\
\hline - Beclometasona & & $200-400$ & $>400$ \\
\hline - Budesonide & $200-400$ & $400-800$ & $>800$ \\
\hline - Fluticasona & $100-250$ & $250-500$ & $>500$ \\
\hline - Mometasona & $110-220$ & $220-440$ & $>440$ \\
\hline \multicolumn{4}{|c|}{ Niños de 6-11 años } \\
\hline - Beclometasona & $50-100$ & $100-200$ & $>200$ \\
\hline - Budesonide & $100-200$ & $200-400$ & $>400$ \\
\hline - Fluticasona & $100-220$ & $200-500$ & $>500$ \\
\hline - Mometasona & 110 & $220-440$ & $>440$ \\
\hline \multicolumn{4}{|c|}{ Niños $\leq 5$ años } \\
\hline - Budesonide & \multicolumn{3}{|c|}{ no recomendad en esta edad } \\
\hline - Fluticasona & 100 & 200 & - \\
\hline - Mometasona & 110 & 220 & - \\
\hline
\end{tabular}

Adaptada de: Global Initiative for Asthma. Global Strategy for Astham Management and Prevention, 2018. 
Tabla No. 4: Pasos de tratamiento.

\begin{tabular}{|c|c|c|c|}
\hline PASO & RESCATE & PRIMERA OPCIÓN & ALTERNATIVA \\
\hline 1 & SABA & & considerar CEI DB \\
\hline 2 & SABA & CEI DB & Antileucotrieno( $<5$ años) \\
\hline 3 & $\begin{array}{l}\text { SABA o } \\
\text { TERAPIA SMART* }\end{array}$ & $\begin{array}{l}>5 \text { años CEI DB + LABA } \\
<5 \text { años CEI DM }\end{array}$ & $\begin{array}{l}\text { CEI DM } \\
\text { CEI DB + montelukast }\end{array}$ \\
\hline 4 & $\begin{array}{l}\text { SABA o } \\
\text { TERAPIA SMART }\end{array}$ & $\begin{array}{l}>5 \text { años CEI DM + LABA } \\
<5 \text { años CEI DM + } 1 \text { o } 2 \\
\text { controladores }\end{array}$ & $\begin{array}{l}\text { + tiotropio/montelukast } \\
\text { O CEI DA }\end{array}$ \\
\hline 5 & $\begin{array}{l}\text { SABA o } \\
\text { TERAPIA SMART }\end{array}$ & $\begin{array}{l}>5 \text { años CEI DA + LABA + } \\
\text { tiotropio (adolescente) }\end{array}$ & CE oral, omalizumab u otro \\
\hline
\end{tabular}

CE: corticoesteroide, CEI: corticoesteroide inhalado, DB: dosis baja, DM: dosis media, DA: dosis alta, LABA: b2-agonistas de acción prolongada, SABA: b2-agonistas de acción corta.

*TERAPIA SMART: CEI DB + formoterol como controlador y rescate.

Adaptada de: Linnemann D, Salas, Hernández J, et al. Mexican Asthma Guidelines: GUIMA 2017 Guía Mexicana del Asma: GUIMA 2017. Rev Alerg Mex Guía Mex del Asma Rev Alerg Mex. 2017;64(1):11-128.

Tabla No. 5: Clasificación de Severidad de la Crisis Asmática en $>6$ años.

\begin{tabular}{|l|l|l|l|}
\hline & \multicolumn{1}{|c|}{ LEVE } & \multicolumn{1}{c|}{ MODERADA } & \multicolumn{1}{c|}{ GRAVE } \\
\hline Disnea & Leve & Intensa & Muy intensa \\
\hline Habla & Párrafos & Frases /palabras & No habla \\
\hline Cianosis & Ausente & Presente & Presente \\
\hline Frec. Respiratoria & Elevada & $>25 \mathrm{rpm}$ & \multicolumn{1}{c|}{-} \\
\hline Frec.Cardiaca & $\begin{array}{l}<100 \\
\text { L/min }\end{array}$ & $100-120 \mathrm{~L} / \mathrm{min}$ & $>120 \mathrm{~L} / \mathrm{min}$ o bradicardia \\
\hline $\begin{array}{l}\text { Uso de músculo } \\
\text { accesorios }\end{array}$ & Ausente & Presente & $\begin{array}{l}\text { Disociación toraco- } \\
\text { abdominal }\end{array}$ \\
\hline Sibilancias & Presentes & Presentes/reducidas & Silencio auscultatorio \\
\hline Nivel de consciencia & Normal & Agitación & Somnolencia \\
\hline FEV1 o PEF & $33-60 \%$ & $25-33 \%$ & $<25 \%$ \\
\hline SatO2 $(\%)$ & $90-95 \%$ & $88-90 \%$ & $<90 \%$ \\
\hline PaO2 $(\mathrm{mmHg})$ & $>80$ & $60-80$ & $<60$ \\
\hline pH & Normal & Puede estar $>7.45$ & $<7.35$ \\
\hline
\end{tabular}

Adaptada de: Linnemann D, Salas, Hernández J, et al. Mexican Asthma Guidelines: GUIMA 2017 Guía Mexicana del Asma: GUIMA 2017. Rev Alerg Mex Guía Mex del Asma Rev Alerg Mex. 2017;64(1):11-128. 
Tabla No. 6: Puntaje de evaluación pulmonar (Pulmonary score). Valoración clínica de la crisis asmática.

\begin{tabular}{|c|c|c|c|c|}
\hline \multirow[b]{2}{*}{ Puntuación } & \multicolumn{2}{|c|}{ Frecuencia Respiratoria } & \multirow{2}{*}{ Sibilancias } & \multirow{2}{*}{$\begin{array}{l}\text { Uso de músculo } \\
\text { esternocleidomastoideo }\end{array}$} \\
\hline & $\leq 6$ años & $\geq 6$ años & & \\
\hline 0 & $<30$ & $<20$ & No & No \\
\hline 1 & $31-45$ & $21-35$ & Final de espiración & Incremento leve \\
\hline 2 & $46-60$ & $36-50$ & Toda la espiración & Aumentado \\
\hline 3 & $>60$ & $>50$ & $\begin{array}{l}\text { Inspiración y } \\
\text { Espiración }\end{array}$ & Actividad máxima \\
\hline & & & $\begin{array}{c}\text { (Sin necesidad de } \\
\text { estetoscopio) }\end{array}$ & \\
\hline
\end{tabular}

\begin{tabular}{|c|c|c|}
\hline Puntaje & Severidad & SatO2 (con oxímetro de pulso) \\
\hline $0-3$ & Leve & $>94 \%$ \\
\hline $4-6$ & Moderado & $91-94 \%$ \\
\hline$\geq 7$ & Grave & $>91 \%$ \\
\hline
\end{tabular}

* Crisis grave: se recomienda hospitalizar

Adaptada de: Linnemann D, Salas, Hernández J, et al. Mexican Asthma Guidelines: GUIMA 2017 Guía Mexicana del Asma: GUIMA 2017. Rev Alerg Mex Guía Mex del Asma Rev Alerg Mex. 2017;64(1):11-128.

\section{REFERENCIAS BIBLIOGRÁFICAS}

1. Larenas Linnemann DES, del Río Navarro BE, Luna Pech JA, et al. Recommendations for the prevention and diagnosis of asthma in children: Evidence from international guidelines adapted for Mexico. Allergol Immunopathol (Madr). 2018;46(3): 291-303.

doi:10.1016/j.aller. 2017.05.011.

2. Lenney W. The burden of pediatric asthma. Pediatr Pulmonol. 1997; 24 (SUPPL. 15):13-16. doi: 10.1002/ (SICI) 1099-0496(199709)15+ <13::AID-PPUL4 $>3.0 . \mathrm{CO} ; 2-\mathrm{S}$.

3. Gallardo Martínez G, Cruz AA, González Díaz SN, Rodríguez GG. Costos derivados de la atención médica del asma en un grupo de niños del Noreste de México. Rev Alerg México Vol Rev Alerg México. 2007;5454(3):82-85.
4. Global Iniciative for Asthma. Global Strategy for Asthma Management and Prevention, 2018. available from:

www.ginasthma.org

5. Linnemann D, Salas, Hernández J, et al. Mexican Asthma Guidelines: GUIMA 2017 Guía Mexicana del Asma: GUIMA 2017. Rev Alerg Mex Guía Mex del Asma Rev Alerg Mex. 2017; 64 (1): 11-128. http://www.revistaalergia.mx.

6. Turner S. The management of childhood asthma - what is new? Paediatr Child Health (Oxford). 2017; 27(7):311-317. doi:10.1016/j.paed.2017.02.008.

7. Hehua Z, Qing C, Shanyan G, Qijun W, Yuhong $Z$. The impact of prenatal expo-sure to air pollution on childhood wheezing and asthma: A systematic 
review. Environ Res. 2017; 159 (36): 519530. doi:10.1016/j.envres.2017.08. 038.

8. Quirt J, Hildebrand KJ, Mazza J, Noya F, Kim H. Asthma. Allergy, Asthma Clin Immunol. 2018;14(S2):50. doi:10.1186/ s13223-018-0279-0.

9. Caillaud D, Leynaert B, Keirsbulck M, et al. Indoor mould exposure, asthma and rhinitis: Findings from systematic reviews and recent longitudinal studies. Eur Respir Rev. 2018; 27(148). doi:10.1183/16000617.0137-2017.

10. Westergren T, Berntsen S, Ludvigsen MS, et al. Relationship between Physical Activity Level and Psychosocial and Socioeconomic Factors and Issues in Children and Adolescents with Asthma: A Scoping Review Protocol. Vol 15.; 2017. doi:10.11124/JBISRIR-2016-0029 45.

11. Xu Z, Crooks JL, Davies JM, Khan AF, Hu $\mathrm{W}$, Tong $\mathrm{S}$. The association between ambient temperature and childhood asthma: a systematic review. Int J Biometeorol. 2018;62(3):471-481.

doi:10.1007/s00484-017-1455-5.

12. Jartti T, Gern JE. Role of viral infections in the development and exacerbation of asthma in children. J Allergy Clin Immunol. 2017; 140(4):895-906.

doi:10.1016/j. jaci.2017.08.003.

13. Page PM, Broek JL, Bousquet $\mathrm{J}$, et al. Global Strategy For Asthma Management and Prevention. Glob Initiat Asthma. 2017;126(3): http://ginasthma. org/2017 -gina-report-global-strat.

doi:10.1183/09031936.00138707.

14. Ramamurthy MB. Asthma Mimickers: Approach to Differential Diagnosis. Indian J Pediatr. 2018;85(8): 667-672. doi:10.1007/s12098-017-2558-z.
15. Belhassen M, De Blic J, Laforest L, Laigle $\mathrm{V}$, Chanut-Vogel C, et al. Recurrent Wheezing in Infants A Population-Based Study. Medicine (Baltimore). 2016 Apr; 95(15): e3404.

doi: 10.1097/MD.0000000000003404.

16. Tenero L, Piazza M, Piacentini G. Recurrent wheezing in children. Transl Pediatr. 2016 Jan; 5(1): 31-36 doi: 10.3978/j.issn.2224-4336.2015.12.01.

17. Papi A, Brightling C, Pedersen SE, Reddel HK. Asthma. Lancet. 2017: 783-800. doi:10.1016/S0140-6736(17) 33311-1.

Salviano LD da S, Taglia-Ferre KD, 18. Lisboa S, Costa ACC da, Campos $\mathrm{H}$ da $S$, March M de FP. Association Between Fraction of Exhaled Nitric Oxide and Spirometry Data and Clinical Control of Asthma in Children and Adolescents. Rev Paul Pediatr. 2018; 36(1): 8 . doi: 10.1590/1984-0462/;2018;36;1;00015.

19. Castro-Rodríguez JA, Holberg CJ, Wright AL, Martinez FD. A Clinical Index to Define Risk of Asthma in Young Children with Recurrent Wheezing. Am J Respir Crit Care Med. 2000 Oct;162(4 Pt 1):1403-6. https://doi.org/10.1164/ajrccm.162.4.991211120. Chang TS, Lemanske RF, Guilbert TW, et al. Evaluation of the Modified Asthma Predictive Index in High-Risk Preschool Children. J Allergy Clin Immonological Pract. 2013; 1(2): 1-12. doi:10.1016/j.jaip.2012.10.00 8 .

20. Bao Y, Chen Z, Liu E, Xiang L, Zhao D, Hong J. Risk Factors in Preschool Children for Predicting Asthma During the Preschool Age and the Early School Age: a Systematic Review and Meta-Analysis. Curr Allergy Asthma Rep. 2017; 17(12). doi:10.1007/s11882 -017-0753-7.

21. Castro-Rodriguez JA. The Asthma Pre dictive Index: A very useful tool for predic 
ting asthma in young children. Allergy Clin Immunol. 2010 Aug; 126(2): 212-6. doi: 10.1016/j.jaci.2010.06.032.

22. Mulholland A, Ainsworth A, Pillarisetti N. Tools in Asthma Evaluation and Management: When and How to Use Them? Indian J Pediatr. 2018;85(8):651-657. doi:10.1007/s12098-017-2462-6.

23. Dweik RA, Boggs PB, Erzurum SC, et al. An official ATS clinical practice guideline: interpretation of exhaled nitric oxide levels (FENO) for clinical applications. Am J Respir Crit Care Med 2011 Sep 1;184( 5): 602-15. doi: 10.1164/rccm.9120-11ST.

24. Malinovschi A, Fonseca JA, Jacinto $T$, et al. Exhaled nitric oxide levels and blood eosinophil counts independently associate with wheeze and asthma events in National Health and Nutrition Examination Survey subjects. J Allergy Clin Immunol 2013 Oct;132(4):821-7.e1-5. doi: 10.1016/j.jaci.2013.06.007

25. Mirra V, Montella S, Santamaria F. Pediatric severe asthma: A case series report and perspectives on anti-IgE treatment. BMC Pediatr. 2018;18(1): 1-11. doi:10.1186/s12887-018-1019-9.

26. Louisias M, Phipatanakul W. Managing Asthma in Low-Income, Underrepresented Minority, and Other Disadvantaged Pediatric Populations: Closing the Gap. Curr Allergy Asthma Rep. 2017;17(10). doi:10.1007/s11882-017-0734-x.

27. Abrams EM, Becker AB, Szefler SJ. Current State and Future of Biologic The rapies in the Treatment of Asthma in Children. Pediatr Allergy Immunol Pulmonol. 2018;31(3):119-131.

doi: 10.1089/ped.2018.0901.

28. Kaur S, Singh V. Asthma and Medicines Long-Term Side-Effects, Monitoring and
Dose Titration. Indian J Pediatr. 2018; 85(9): 748-756.

doi:10.1007/s12098-017-2553-4.

29. Gionfriddo MR, Hagan JB, Rank MA. Why and how to step down chronic asthma drugs. BMJ. 2017;359:1-12. doi:10.1136/bmj.j4438.

30. Geryk LL, Roberts CA, Carpenter DM. A systematic review of school-based interventions that include inhaler technique education. Respir Med. 2017;132:21-30. doi:10.1016/j.rmed.2017.09.001.

31. Barbara S, Kritikos V, Anticevich SB. Inhaler technique: Does age matter? A systematic review. Eur Respir Rev. 2017;26(146):1-10.

doi:10.1183/16000617.0055-2017.

32. Katwa U, Rivera E. Asthma Management in the Era of Smart-Medicine: Devices, Gadgets, Apps and Telemedicine. Indian $J$ Pediatr. 2018;85(9): 757-762. doi:10.1007/s12098-018-2611-6.

33. Normansell R, Sayer B, Waterson S, Dennett EJ, Del Forno M, Dunleavy A. Antibiotics for exacerbations of asthma. Cochrane Database Syst Rev. 2018; 2018(6).

doi:10.1002/14651858.CD002741.pub2.

34. Magpuri AT, Dixon JK, McCorkle R, Crowley AA. Adapting an Evidence-Based Pediatric Acute Asthma Exacerbation Severity Assessment Tool for Pediatric Primary Care. J Pediatr Heal Care. 2018;32(1):10-20.

doi:10.1016/j.pedhc.2017.06.004.

35. Anderson WC, Gleason MC, Miyazawa N, Szefler SJ. Approaching Current and New Drug Therapies for Pediatric Asthma. Pediatr Clin North Am. 2017; 64 (6): 1197-1207. doi:10.1016/j.pcl.2017.08.001. 